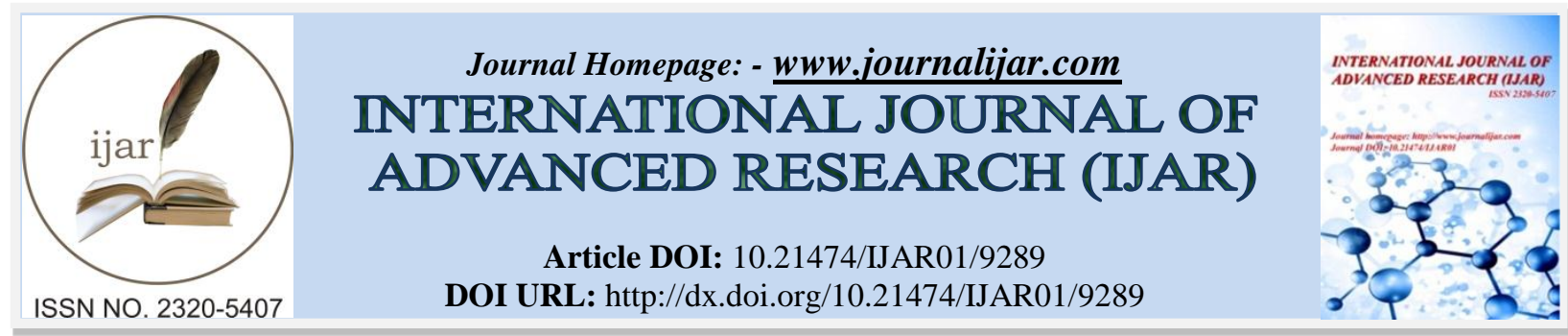

RESEARCH ARTICLE

\title{
LES REVERIES D'UN ELDORADO: REFLEXIONS SUR LES MESAVENTURES D'AMOIN DANS PAIN SUCRE DE MARY LEE MARTIN-KONE.
}

Ebong, Offiong Erete ${ }^{1}$ and Malumi, Soni Omoloro ${ }^{2}$.

1. Department of modern languages and translation studies, university of calabar.

2. Department of foreign languages, university of jos.

\section{Manuscript Info}

Manuscript History

Received: 15 April 2019

Final Accepted: 17 May 2019

Published: June 2019

\begin{abstract}
Cette communication examine la réalité du rêve d'une villageoise africaine dans une société dominée entièrement par l'homme. Tout le monde est libre d'aspirer d'être une personnalité célèbre dans la vie. Dans cette communication nous analyserons l'aspiration bafouée d'une villageoise africaine qui avait rêvé de faire la carrière dans la chanson, et puis devient une grande chanteuse et puis sortir un disque. C'est ce désir de réussir à tout prix qui l'a poussé d'aller en ville précisément dans la capitale de son pays. Le contact de notre protagoniste «Amoin » avec le monde extérieur hors de son tranquille village a été pathétique. Elle a été confrontée par la dure réalité de la vie humaine. Au lieu d'être une personnalité célèbre comme rêvait, elle finit par être une vendeuse de pain sucré. Ce travail devrait donc permettre de mettre en évidence non seulement l'aspiration bafouée d'une villageoise africaine mais devrait permettre aussi d'analyser l'œuvre de Mary Lee Martin-Koné «pain sucré » en mettant en lumière les mésaventures qui suivent l'aspiration d'une jeune fille en Afrique. Donc, notre objectif principal est de décourager les jeunes villageois et villageoise qui voient la métropole comme le paradis souhaité, comme un Eldorado où il faut mieux vivre d'abandonner l'idée d'y voyager à tout prix et lutter pour son épanouissement dans son village natal.
\end{abstract}

Copy Right, IJAR, 2019,. All rights reserved.

\section{Introduction:-}

Le Tout puissant a donné la liberté à chacun de nous d'aspirer, de rêver et de désirer la meilleure dans la vie. Malgré son niveau d'éducation, notre protagoniste dans Pain Sucré «Amoin » espère toujours trouver un Eldorado qui pousse combler ses rêves et ses attentes, elle a sacrifié son temps et son argent pour être dans son Eldorado souhaité. Le désir d'aller réaliser son rêve en ville a été tronqué. C'est ce qui se passe actuellement avec nos jeunes nigérians d'aujourd'hui qui voulaient aller en Europe à tout prix. Espérant que, l'Europe offira la meilleur dans la vie. Beaucoup d'eux finissent par être tués soit sur la mer rouge ou en arrivée en Europe. Le désir d'aller en ville à tout prix est derrière le rêve bafoué de plupart de nos jeunes nigérians d'aujourd'hui. Toujours derrière cette mis fortune des jeunes est l'homme. Mais avant d'entrer dans le vif de cette analyse, jetons un coup d'œil sur les termes qui portent le drapeau de notre travail afin de savoir la direction de notre analyse. Les trois termes qui méritent une explication sont «rêverie », «Eldorado » et mésaventures. D'après Angrey, le mot «rêverie » est dérivé du mot «rêve» qui est «la suite de phénomènes psychiques se produisant pendant le sommeil ou » la construction imaginaire destinée à échapper au réel, à satisfaire un désir, à refuser une réalité pénible. A travers cette explication, 
nous pouvons dire qu'on rêve lorsqu'on dort qui est une suite d'images qui se présentent à l'esprit pendant le sommeil. Dans ce cas pour rêver il faut tout d'abord dormir. Le rêve dans cette situation ne vient qu'avec le sommeil. D'une autre manière, on peut rêver au niveau physique sans dormir. Dans ce cas « rêve » s'applique aux désirs que l'on formule. Donc, «rêverie » dérivé du mot «rêve » est un état. C'est l'état de l'esprit qui s'adonne à des images vagues sans chercher à en modifier le cours. L'explication de rêverie que nous venons de voir nous apprend que ce mot souligne l'état d'âme de quelqu'un. L'homme s'adonne à certaines idées, à certaines images imprécises. Le résultat en est que l'homme finit par ne rien gagner de ses rêveries. Il est presqu' impuissant devant son destin dont il est incapable de changer le cours.

A propos du mot «Eldorado » qui est un terme détourné de son origine mythologique est utilisé pour designer une contrée fantasmée où ses habitants vivent et s'épanouissent dans l'abondance et l'allégresse. C'est un lieu de vie facile. Eldorado était un pays imaginaire que les conquistadors espagnols pensaient découvrir entre l'Amazone et l'Orénoque. Ils croyaient trouver une multitude de trésors qui leur rendraient la vie facile. C'est de la que provient le nom « Eldorado » que l'on utilise pour designer un lieu où la vie est facile ; un lieu d'opportunité où il faux mieux vivre, un lieu où il faut visiter à tout prix, un lieu de bénédiction.

De sa part le mot «mésaventure » peut se voir comme un événement triste, une aventure fâcheuse, désagréable qui arrive à quelqu'un. C'est un moment d'angoisse et de lamentation qui peut détruire n'importe quelle personne sur terre. C'est un moment ou l'on perd l'espoir de la vie.

Les rêveries d'un Eldorado se manifestent dans le roman d'étude à travers le personnage d'Amoin qui avait rêve de faire la carrière dans la chanson. Son espoir, son aspiration et son désir a été détruite. Elle finit par être une vendeuse de pain sucré.

\section{Que nous presente la romanciere et le roman d'etude « pain sucre »}

Née aux États-Unis, Mary Lee Martin-Koné y passe son enfance avant de partir en Côte d'ivoire de 1977 à 1990 où elle enseigne l'anglais. Elle a déjà publié un merveilleux conte moderne : La Force du vouloir. Mariée à l'écrivain Amadou Koné, elle est également mère de quatre enfants. Après avoir résidé en Allemagne, elle vit à nouveau depuis 1996 aux États-Unis.

Pain sucré est un roman de 125 pages écrit en 2002. Le roman s'agit d'une jeune fille qui s'appelle Amoin qui voulait profiter de ses vacances pour réaliser ses rêves d'être artiste à l'image d'une grande artiste appelée Fifi Franco qu'elle considère comme son idole. Une invitation de sa tante à passer quelque semaine de vacances en ville l'entraine à échafauder mille projets, mais un concours de circonstances imprévues fait qu'elle tombe entre les mains d'un marchand cupide de fillettes. Retenue contre son gré par le maître impitoyable, elle devient la vendeuse de pain sucré au lieu de la carrière qu'elle avait choisi et puis elle est devenue domestique chez la téléspeakerine de la «k.k.m variétés». Amoin a toutes les peines du monde à retourner à la liberté. Elle finit par regretter sa décision d'avoir quitter son tranquille village à cause d'un rêve d'être une artiste célèbre qui est devenue un cauchemar pour elle.

\section{L'image De La Metropole Chez Les Jeunes Villageois Africains}

Les jeunes villageois ont une image assez positive de la métropole. Cela montre que certains jeunes villageois ont une connaissance assez approfondie de la ville et son état de développement ; ces savoirs emmagasinés alimenteront le désir d'aller en ville à tout prix. Nous trouvons cet amour d'Amoin pour la métropole à travers son dialogue avec son amie :

Dans la ville, c'est la belle vie, dit-elle. Tout ce qu'on voit là-bas est beau. Il ya des maisons à étages qui montent jusqu'au ciel. Il ya des hôtels de luxe avec leur piscine, des cinémas climatisés, des boutique de prêt à-porter. Je vais revenir avec ces robes... je vous assure (28).

Avant même d'avoir vu la métropole, l'idée que la ville est supérieure au village est déjà plantée dans les cerveaux d'Amoin. Même avant d'aller, son esprit est déjà dans la ville :

Si tu crois que je vais rester ici pour assister à un petit mariage villageois, tu as tort. Moi, j'irai plutôt vois de grands mariages, à l'hôtel de ville, avec la mariée dans sa robe blanche, et son mari dans sa redingote. J'assisterai à des mariages avec beaucoup de fleurs, des photographes, la télévision, des défiles de grosses voitures (30). 
Comme nous venons de signaler l'admiration de la métropole chez cette jeune villageoise commence bien avant son arrivée dans cette métropole. Avant même d'avoir voyagé hors de son village natal, Il ya déjà dans la tête d'Amoin que la ville est un Eldorado, un paradis souhaité où il faut mieux vivre. Nous avons vu cet amour profonde pour la métropole à travers cette parole d'Amoin :

Chez Tonton. C'est ultra-moderne. Il habite au septième étage d'un grand immeuble. On prend l'ascenseur pour y'monter. L'ascenseur, il paraît que c'est un peu comme l'avion ... chez Tonton, Il ya tout, tout ! Frigidaire, cuisinière, climatiseurs, téléviseur couleur, chaîne stéréo, de grands lit bien confortables. Ce n'est pas comme ici au village, où on dort par terre ; dans la chaleur, où on mange mal, où on se feu à travailler sans jamais s'amuser, ici où il ne se passe jamais rien (28).

Malgré le fait qu'elle n'a jamais visité la capitale, le village n'a rien à offrir. La place idéale pour réussir et pour être une personnalité célèbre est la métropole. Voila pour qu'elle n'est pas prête d'être découragé par son amie. Avec son projets dans sa tête, notre protagoniste était très sûre d'accomplir son rêve dans la métropole.

De la même façon, Innocent dans pris au piège avait rêvé d'un Eldorado en ville. Il avait eu l'opportunité d'y aller grâce à une invitation de son oncle après son succès au certificat d'études primaires. Son arrivée chez son oncle était marqué par la haine et la jalousie à cause de son intelligence d'abord de l'épouse, puis de l'oncle. Les deux lui feront toutes les misères possibles jusqu' à l'expulser de leur villa pour de sombres raisons. Le sentiment de jalousie est perçu à travers son oncle et sa femme. En effet après avoir annoncé son succès en classe supérieure Innocent surprend son oncle et sa femme en plein discussion :

Te rends-tu compte comme moi qu'Innocent depuis le matin, où il a annoncé son passage en classe supérieure semble se moquer de ses cousins, remarqua mon père adoptif. Mon propre oncle. Je vois qu'à présent, tu commences par comprendre. Je te l'avais dit une fois qu'il était entré dans cette maison, il est impoli et ingrat, renchérit la femme (54).

Innocent qui avait rêvé d'un Eldorado avant son départ pour la capitale économique est devenu un enfant de la rue, son rêve prometteur a été tronqué. Il finit par retourner au village est il a beaucoup regretté son séjour dans la métropole.

Dans Un Nègre à Paris, Bértin avant son voyage à Paris avait une image positive de cette métropole. Il avait beaucoup rêvé de cette métropole. Nous avons vu son amour profonde pour paris à travers sa parole à son ami :

La bonne nouvelle, mon ami ! la bonne nouvelle! j'ai un billet pour paris, oui, paris ! Paris dont nous avons toujours tant parlé, tant rêve. Je vais voir paris, moi aussi ; avec mes yeux. Désormais, je serai un peu comme tout le monde ... je vais toucher les murs, les arbres, croiser les hommes (1).

Mais son contact avec la métropole a changé sa vision et son image de cette ville. Il a constaté que, la ville de Paris est un lieu de désillusionnement. Voila comment il nous décrit la métropole. «Paris est un monde ... un océan dans lequel on risque de se noyer si l'on ne sait pas nager »(16).

Bertin est venu à paris avec pleins de rêves, d'illusions et de projets mais il était choqué de voir la ségrégation raciale, l'exploitation de l'homme par l'homme, la dégradation et la débauche à Paris. Il annonce immédiatement son retour au pays natal malgré le fait qu'il n'est pas entièrement parvenu à son but : moi aussi, il faut que je parte sans avoir pu, hélas ! Tout voir ... (216).

Le comportement de Bertin nous montre que si paris représentait un rêve de paradisiaque, un Eldorado, à son contact, ce paradis se transformait en enfer.

Dans le cas de Samba Diallo héros au pays des Diallobé, il avait rêvé d'aller à paris, il a eu l'opportunité d 'y aller pour étudier la philosophie. Mais son contact avec le monde extérieur a changé sa vision de vie. Il est revenu au pays natal désaxé et puis il a trouvé sa mort prématuré dans la main du fou. 
De la même façon dans L'Enfant noir, Camara Laye nous montre le rêvé de paris par un enfant noir depuis des années. Il a beaucoup rêve de cette métropole mais un jour il a eu l'opportunité d'y aller grâce a une bourse scolaire. «Et je me mis à rêver à paris : Il y avait tant d'années qu'on me parlait de Paris » (175), mais sa perception de cette métropole a changé à son arrivée. Avant son départ, il avait rêvé d'un paradis, d'un Eldorado, d'une vie nouvelle mais son contact avec ce monde extérieur a changé sa vision de paris. Le narrateur promet de revenir au pays natal mais son dernier geste est de palper le plain du métro de paris qui gonfle sa poche.

De la même façon Alain Mabanckou dans Black Bazar nous montre l'horreur de la métropole de paris à travers le personnage de Fessologue qui est venu à Paris avec pleins de projets et rêves mais ces rêves se terminent au cauchemar. Les rêves d'un Eldorado, d'un lieu où il faut mieux vivre a été tronqué à cause de la couleur d'origine. Il finit par regretter son rêve de venir à Paris.

Dans mirages de Paris d'Ousmane Socé, chemin d'Europe de Ferdinand Oyono et Kocoumbo d'Aké Loba l'histoire reste les mêmes. Venus à Paris avec pleins de projets, d'illusions. Les personnages principaux vont très vite déchanter, passée l'euphorie de l'annonce du départ, et des premiers instant à paris, ils vont être pour certains emporter par un tourbillon négatif matérialisé par un changement de mentalité ni le vulgaire. Le dispute au ridicule Paris exerce-t-elle sur eux une influence perverse.

Nous avons constaté que, les rêveries d'un Eldorado par nos jeunes villageois de la métropole soit en Afrique ou en Europe a été un cauchemar. Avant de partir pour la ville, ils ont une image positive de réussir à tout prix mais le contact avec ce monde extérieur se transformait en vrai enfer.

\section{Les reves de gloire et les mesaventures d'amon dans la capitale.}

Le protagoniste dans Pain Sucré, Amoin avait rêve de quitter son tranquille village pour se rendre à la grande ville dans le but de faire carrière dans la chanson, sortir un disque et entrer dans un concours de danse comme elle nous rêve le à travers son dialogue avec Rose :

Entrer dans un concours de danse n'est qu'un tremplin pour moi. Je vise plus hait que de remporter un concours de danse. J'espère surtout être remarquée par un chanter ayant besoin de danseuses et plus tard un imprésario. Je compte surtout entrer en contact avec des gens important de la télévision comme Frédéric Kamaté, Celui qu'on appelle «F. K., M. variétés ». Je suis certain que tous ceuxlà m'aideront à le lancer dans ce merveilleux monde de la chanson. Vraiment je crois que la route du succès ne va pas tarder à s'ouvrir devant moi (11).

Pour réaliser ce rêve d'un Eldorado, Amoin compte voyager à la capitale. Elle pense qu'en arrivée à la capitale, l'histoire de sa vie changera complètement et son rêve d'un Eldorado sera réaliser à travers cette parole.

La capitale est un cadre plus favorable aux deux activités. Pour les études, je serai chez mon oncle qui est professeur lui-même et qui pourra bien m'encadrer. Pour la carrière de l'artiste c'est l'endroit idéal car il ya toutes les infrastructures: Imprésario, studio d'enregistrement, salle des fêtes. C'est le paradis (13).

Pendant les grandes vacances, le rêve de la jeune fille de voyager à la capitale a été réaliste. Son oncle qui est professeur et sa femme lui a invité de venir passer ses vacances chez eux. Son oncle lui a demandé d'arriver à la gare routière de la ville à deux heures de l'après-midi, mais notre amie Amoin n'a pas honoré le rende vous. Au lieu d'arriver à la gare routière de la ville à deux heures elle est arrivée au crépuscule à cause des raisons techniques et logistiques.

Son oncle est venu lui attendre à deux heures de l'après-midi à la gare routière mais Amoin était toujours sur la route. C'est à ce moment précis que commence la souffrance de la jeune fille.

Comme son oncle est déjà partie sans voir sa nièce, Amoin est tombée dans la main d'une vendeuse de Pain Sucré que est petite comme elle :

Alors, Amoin s'approche de la dernière vendeuse de Pain Sucré qui est en train de plier ses affaires pour rentre chez elle. Amoin s'achète un petit pain et e met à 
la manger goulument. Comme la vendeuse est petite comme elle cela encourage Amoin à lui parler (37).

C'est grâce à cette vendeuse de Pain Sucré qu'Amoin a eu le renseignement sur son oncle : Oui, j'ai bien vu un homme ça ici viens deux heures. Il a attendu longtemps sans voir personne. Fatigué, il est parti (37).

Avec un rende vous raté, Amoin est à la merci d'Adèle, la vendeuse de Pain Sucré. Adèle demande à Amoin de venir passer la nuit chez elle comme elle n'a pas d'argent et elle ne sait pas où elle va dormir. Cette invitation marque le début de l'exploitation de la petite villageoise qui décide de venir à la capitale pour réaliser son rêve musicale.

Le deuxième jour, son oncle est revenu à la gare routière pour la chercher mais à cause de l'action diabolique d'Adèle, il n'a pu la voir. Que fait une villageoise dans cette circonstance? Sans l'argent pour repartir à son village et sans avoir aucun renseignement concernant son oncle. Donc, son dernier espoir est sur Adèle et son oncle. Pour Amoin, il n'y a qu'un seul recours : La seule personne qui peut l'aider, c'est le père d'Adèle, Elle ne connait personne d'autre (58).

Mais son rende vous avec le père d'Adèle relève la méchanceté dans le cœur de l'homme. Amoin demande à cet homme de lui prêter l'argent pour retourner au village mais sa réponse révèle sa personnalité et sa méchanceté :

Ma petite, le crédit est mort. Est-ce ma faute si tu n'as pas prévu assez d'argent quand tu venais ici ?... maintenant, tu veux que je le donne de le donne de l'argent pour reparti. Tu a vu la multitude de personnes qui vivent déjà ici? (62).

Amoin était ébahie avec cette proposition et pour conclure le père d'Adèle dit : D'abord, tu vas rembourser le prix de la nourriture que tu consommes depuis ton arrivée. Ensuite, il faudra que tu gagnes toi-même le prix du taxi pour retourner dans ton village (62).

C'est à ce moment précis que, la jeune fille Amoin qui a rêvé d'avoir un moment inoubliable dans la capitale chez son oncle a été initiée dans le business de la vendeuse de Pain Sucre. Elle est devenue en même temps une jeune fille sans liberté, une jeune fille perdue, une jeune fille à la merci d'un vieillard et d'une fille qu'elle ne connait pas comme explique Adèle :

A partir de demain, plus de gras matinée pour toi, il faudra te lever avant le lever du jour pour m'aider à cuire le pain et puis c'est toi qui iras à la gare vendre le pain à ma place (63)

Le vieillard ajoute que, si Amoin ne travaille pas bien, la chicotte est à la disponibilité d'Adèle. Avec cette affirmation, Amoin est devenue un animal sauvage.

A la fin de ce rende vous hostile avec Adèle et son oncle, Amoin était confrontée avec la dure réalité de la vie comme explique la narratrice : Elle n'a jamais été aussi abattue de sa vie : ses yeux pleins de larmes, le cœur angoissé. Elle se tourne et se tourne sans trouver le sommeil (63).

Afin de trouver de l'argent pour retourner chez elle, Amoin finit par être intégrée dans le business de la vendeuse de pain sucré. Amoin est traitée comme un animal par son patron, si par hasard, elle revient sans vendre tous les pains donnés comme explique la narratrice la narratrice, le ciel va tomber: Pas assez de monde ! Hurle le vieux prends ça, dit il subitement en donnait plusieurs coups de chicote à Amoin (69).

Avec toutes les souffrances, Amoin aimerait fuir de la cour, mais où va-t-elle ? Elle est devenue une prisonnière chez un vieillard qu'elle ne connait pas. Il est à noter que, ce n'est pas seulement Amoin qui est victime de cette mésaventure chez cet homme diabolique comme explique la narratrice : «De nombreuses fillette sont couchées dans tous les sens et à même le sol (59) »

Amoin et les autres jeunes filles chez l'oncle d'Adèle sont toujours battues, molestées, maltraitées et malmenées. Le vieux c'est-à-dire, le père d'Amoin va dans les villages pour convaincre les jeunes filles de venir en ville comme explique la narratrice : 
Quelque fois même le vieux va dans les villages pour recruter les filles trop contentes de quitter leur village. Elles travaillent pour lui sans salaire et de temps en temps s'il veut, il leur achète quelques vêtements ou des chaussures (80).

La proposition d'Antoinette nous révèle le caractère diabolique de l'oncle d'Adèle :

Moi, je connais ce vieux mieux que toi. Tous les jours, tu lui apportes de l'argent et tu penses qu'il rate lâcher si facilement? Quand tu penseras que ta dette est payée. Il va te garder ici le plus longtemps possible pour pouvoir t'exploiter au maximum (82).

Avec toute cette révélation, Amoin a décidé de s'enfuir. Elle a tout fait pour s'enfuir mais cela n'était pas réalisée. Elle était attrapée à la gare routière par Adèle. L'oncle d'Adèle était très furieux en apprenant cette nouvelle et Amoin était enfermée dans une case obscure comme une punition :

Le vieux rentre dans sa chambre et en ressort aussitôt avec un trousseau de grosses clefs. Il se dirige au fond de la cour vers une petite case dont il ouvre la porte. Ill demande ensuite à Adèle et à l'homme de transporter Amoin dans cette case. Quand Amoin comprend leur intention, elle se remet à se débattre furieusement. Mais elle est vite maitrisée et j'étée dans la petite pièce obscure. Le vieux refreine la porte à clef sans tenir compte des hurlements et des pleurs d'Amoin (92).

Amoin devient une prisonnière chez un vieillard qu'elle n'a pas connu. Elle était finalement libérée, un jour après avoir subi une punition terrible. A cause de cette action, Amoin était surveillée partout par Adèle. Donc, elle a été envoyée au quartier des deux collines comme bonne cuisinière avec Adèle comme sa patronne. C'est chez cette dame qu'elle a trouvé son oncle.

C'est les mésaventures qui suivent les rêvés de gloires dans la métropole. Le rêve d'une jeune fille étant tronquée Amoin est devenue misérable dans la ville et elle a abandonné sa carrière musicale pour retourner au village à cause d'une action diabolique de l'homme par l'homme.

\section{Conclusion:-}

Dans cette communication, nous avons parlé des rêveries d'un Eldorado par une jeune fille déterminée et ambitieuse dans la capitale de son pays. Cette jeune fille avec un avenir prometteur voulait faire la carrière dans la chansons et puis sortir un disque. Mais son voyage à la métropole pour poursuivre ce rêve révèle que le rêve d'une jeune fille n'est pas facile à réaliser en Afrique. Avant même d'avoir voyagé à la métropole, Amoin a déjà l'image et la conception que, la métropole est le lieu idéal pour réussir dans la vie. Mais son contact avec ce monde extérieur lui montre que, «c'est plus facile à un chameau de passer par le trou d'une aiguille qu' à une jeune fille de réaliser facilement son rêve en Afrique ».

Dans ce continent de l'homme, la voix de la femme est toujours silencée et son avenir est toujours tronquée. Le rêve prometteur d'une jeune fille a été tronquée et ses rêveries d'un Eldorado a été mis à l'eau c'est-à-dire il est devenue un cauchemar. Elle était frustrée et a beaucoup regretté son désir en premier lieu d'avoir nourri l'idée de voyager à la métropole. Il est important de souligner enfin que dans cette communication, nous avons présenté une jeune fille qui rêve d'aller à la métropole, qui est impatiente d'y arriver mais qui est totalement déçue et découragée à son contact avec la métropole à cause de son exploitation physique. Elle finit par être une vendeuse de pain sucre au lieu d'être une artiste célèbre comme rêvait.

\section{References:-}

1. Ahmadou, Kourouma. Allah n'est pas obligé, Paris: Editions du seuil, 2000.

2. Angrey, Unimna. Les Rêveries du père dans le roman antillais, Lagos, serenity publishers, 2008.

3. Dadié, Binlin Bernard. Un Nègre à Paris. Présence africaine, Paris 1959.

4. Diop, Ousmane Socé. Mirages de paris : Editions latines, Paris 1977.

5. Gerard, Ake Loba. Kocoumbo, l'étudiant noir, Flammarion, l'université de Virginie, 1960.

6. Kane, Hamidou Cheikh. L'Aventure ambigue Dakar, Juillard, 1961.

7. Laye, Camera. L'Enfant noir.

8. Martin - Koné Mary Lee. Pain Sucre, Paris : Editions Haitier International, 2002. 
9. Oyono Ferdinard. Chemin d'Europe, Paris : René Julliard, 1974.

10. Paul Robert. Dictionnaire Alphabétique et Analogique de la langue francaise, Paris: Nouvelles Editions Millésimes, 2012.

11. Pliya, Jean. La secrétaire particulière, Yaoundé: Editions, CLE, 2001.

12. Tangah, Abdoul-Hammed. Pris au piège, Cotonou : Les Editions Plurielles, 2011.

13. Sitographie: STADTLEDR, Katharina. Regards africains sur la ville de Paris, www.arts.uwa.edu.au/mots pluriels. 